\title{
EXISTENCE THEOREMS FOR A LINEAR PARTIAL DIFFERENCE EQUATION OF THE INTERMEDIATE TYPE*
}

BY

\section{RAYMOND ADAMS}

In a recent paperi the author has called attention to three types of linear partial difference equations constructed by analogy with the two types of difference equations in one independent variable which have already been made the object of a considerable amount of study. To the third of these types, which is in the nature of a cross between the linear ordinary difference equation and the linear ordinary $q$-difference equation, he has ventured to attach the name linear partial difference equation of the intermediate type. It is the following special equation of this type to which the reader's consideration is now invited:

$$
g(x+1, r y)=p(x, y) g(x, y),
$$

where $r$ is a constant, real or complex and different from zero; $p(x, y)$ is a known analytic function of the complex variables $x$ and $y$; and $g(x, y)$ is the function to be determined. This is the equation of first order of the class $\left(C^{\prime}\right)$ spoken of in the paper just now referred to. $\ddagger$ Some study of the equation of order $n$ of this class has been made by the writer, but the comparative simplicity of the theory of the equation of first order in the face of the rather extreme complexity of that of the more general type of equation, and the fact that somewhat more inclusive theorems can be proved for the former than for the latter, have made it seem desirable to present first a treatment of equation (1).

The method employed below is a natural modification of that introduced by Birkhoff $\S$ in his important paper on the linear ordinary difference equation. In $\S 1$ formal series solutions of (1) are found when the coefficient function $p(x, y)$ is a polynomial in $x$ and $y$. In $\S 2$ the existence is established of what may properly be termed principal solutions. Certain extensions of the results of $\S \S 1,2$ are indicated in $\S 3$.

* Presented to the Society, December 30, 1924; received by the editors in June, 1925.

$\dagger$ The general theory of a class of linear partial q-difference equations, these Transactions, vol. 26 (1924), pp. 283-312. This paper will hereinafter be referred to as I.

$\ddagger$ Loc. cit., p. 284. 284.

\$ General theory of linear difference equations, these Trans action s, vol. 12 (1911), pp. 243 - 
It is appropriate to remark here that results similar to those obtained in the following pages can be proved for the equation in $N$ independent variables

$$
g\left(x+1, r_{1} y, r_{2} z, \cdots, r_{N-1} w\right)=p(x, y, z, \cdots, w) g(x, y, z, \cdots, w) .
$$

\section{THE FORMAL SERIES SOlUtions}

It is our purpose to show that there exist analytic solutions of the equation (1) when $p(x, y)$ is a given polynomial

$$
p(x, y)=p+p_{10} x+p_{01} y+\cdots+p_{l m} x^{l} y^{m} .
$$

Two symbolic solutions obtained by iterating (1) are the infinite products*

$$
\begin{aligned}
& p(x-1, y / r) p\left(x-2, y / r^{2}\right) p\left(x-3, y / r^{3}\right) \cdots, \\
& p^{-1}(x, y) p^{-1}(x+1, r y) p^{-1}\left(x+2, r^{2} y\right) \cdots ;
\end{aligned}
$$

certain modifications of these will be the basis of our proof.

Let the degree of $p(x, y)$ in $x$ be $\mu$ and its degree in $y$ be $\nu$. It will be assumed that we have

$$
p_{\mu 0} \neq 0 \quad \text { and } \quad p_{\mu} \neq 0 .
$$

Under these conditions and if $|r|$ is $\neq 1$, it is found by direct substitution and reckoning of the coefficients that equation (1) has two formal series solutions: $\dagger$

(2) $s(x, y)=x^{\mu x} e^{-\mu x} x^{a} y^{p}\left[s+\frac{s_{10}}{x}+s_{01} y+\frac{s_{20}}{x^{2}}+\frac{s_{11} y}{x}+s_{02} y^{2}+\cdots\right]$,

(3) $s^{\prime}(x, y)=x^{\mu-\sigma}-{ }_{-\mu x} \gamma_{\nu}\left(\tau^{2}-\tau\right) / 2 x^{b} y^{\prime}\left[s^{\prime}+\frac{s^{\prime}{ }_{10}}{x}+\frac{s_{01}^{\prime}}{y}+\frac{s_{20}^{\prime}}{x^{2}}+\frac{s_{11}^{\prime}}{x y}+\frac{s_{02}^{\prime}}{y^{2}}+\cdots\right]$,

where

$$
\rho=\frac{\log p_{\mu 0}}{\log r}, \quad \quad \rho^{\prime}=\frac{\log p_{\mu \nu}}{\log r}, \quad \text { and } \quad \tau=\frac{\log y}{\log r} .
$$

- The reciprocal $1 / p(x, y)$ will be denoted by $p^{-1}(x, y)$.

$\dagger$ For the actual calculation of the coefficients in the series (2) and (3) use should be made of the expansion

$$
\left(1+\frac{1}{x}\right)^{\mu x}=e^{\mu x \log (1+1 / x)}=e^{\mu}-\frac{\mu e^{\mu}}{2 x}+\frac{\mu(3 \mu+8) e^{\mu}}{24 x^{2}}-\cdots .
$$

It should be observed that the power of $y$ occurring immediately before the bracketed series in each solution is not a uniquely determined factor; in place of $y^{p}$ in (2), for example, we might use $\sigma^{x}$ as in the case of the linear ordinary difference equation, or in fact, any function $f(x, y)$ such that $f(x+1, r y)=c f(x, y)$, where $c$ is a constant. 
Throughout this paper we shall take the determination of $\log r$ in which the coefficient of $\sqrt{-1}$ is positive or zero and less than $2 \pi$.

In general the formal series solutions (2) and (3) fail to converge; it will be shown, however, that solutions of (1) do exist which are analytic (except perhaps for poles) over the entire finite $x$ - and $y$-planes ( $y=0$ excepted for the second solution), and which are asymptotically represented, in a sense presently to be defined, by the formal series (2) and (3).

The most general analytic solution of (1) is given by

$$
h(x, y)=g(x, y) a(x, y),
$$

where $g(x, y)$ is any particular analytic solution and $a(x, y)$ is any arbitrary analytic function not identically zero and periodic in such a fashion that $a(x+1, r y)=a(x, y)$.

Throughout the remainder of this paper it will be assumed that $|r|$ is greater than unity. If $|r|$ is $<1$, an entirely analogous treatment is possible. In this second case the rôles which the symbolic solutions $p(x-1, y / r) p\left(x-2, y / r^{2}\right) \cdots$ and $p^{-1}(x, y) p^{-1}(x+1, r y) \cdots$ play in the first case are interchanged. The sort of parallelism that occurs is like that which has been found in the author's earlier paper on partial $q$-difference equations, ${ }^{*}$ and has been illustrated so well there as to make it unnecessary for us in this paper to give explicit statements of the theorems when $|r|$ is $<1$.

\section{THE EXISTENCE OF PRINCIPAL SOLUTIONS}

Let $t(x, y)$ denote the polynomial which $s(x, y)$ becomes upon breaking off the bracketed series in (2) so as to retain only terms whose degree in $1 / x$ and $y$ together is less than $k$, or, more generally, a series obtained from $s(x, y)$ by replacing the bracketed series there by one which has the same terms up to and including those of degree $k-1$, but which converges in the vicinity of the place $\dagger(\infty, 0)$. We may then define a function $q(x, y)$ by the relation

$$
t(x+1, r y)=q(x, y) t(x, y) ;
$$

$q(x, y)$ is thus a function of the form $x^{\mu}\left[q+q_{10} / x+q_{01} y+\cdots\right]$ in which the series in brackets has exactly the same terms of degree less than $k$ in $1 / x$ and $y$ as has $x^{-\mu} p(x, y)$. This is seen directly upon comparing

$$
q(x, y)=t(x+1, r y) t^{-1}(x, y) \text { and } p(x, y)=s(x+1, r y) s^{-1}(x, y),
$$

\footnotetext{
* I, pp. 291-298.

$\dagger$ We shall think of the complex variables $x$ and $y$ as represented by points in two distinct planes and shall speak of a pair of associated values of $x$ and $y$ as the place $(x, y)$.
} 
for $t(x+1, r y)$ is the same as $s(x+1, r y)$, and $t^{-1}(x, y)$ the same as $s^{-1}(x, y)$, up to and including terms of degree $k-1$ in $1 / x$ and $y$. It follows that we have

$$
p(x, y)=q(x, y)+m(x, y)
$$

where $m(x, y)$ is a series in powers of $1 / x$ and $y$ whose lowest degree terms are of degree $k$ or higher, and which converges in the vicinity of $(\infty, 0)$. Hence $n(x, y)$, defined by the equation

$$
m(x, y)=q(x, y) n(x, y)
$$

is a power series of the same type as $m(x, y)$, and we have

$$
p(x, y)=q(x, y)[1+n(x, y)]
$$

Before proceeding further with our development it is necessary to make a convenient and not unnatural definition of what we shall mean by asymptotic representation of a function by a series of the type (2). Let $g(x, y)$ be a given function, and consider the difference

$$
g(x, y) x^{-\mu x} e^{\mu x} x^{-a} y^{-\rho}-\left[s+\frac{s_{10}}{x}+s_{01} y+\cdots\right]
$$

where the bracket contains all the terms in $1 / x$ and $y$ up to and including those in $(1 / x)^{s}$ and $y^{t}$ and only those. If for each value of $s$ and of $t$ this difference consists of two parts one of which becomes uniformly small of order $x^{-(s+1)}$ as $x$ becomes infinite in a certain region and the other of which becomes uniformly small of order $y^{t+1}$ as $y$ approaches zero in any manner whatsoever, then we say that $g(x, y)$ is asymptotically represented by $s(x, y)$ in that region with respect to $x$, for $|y|$ small; if the first part becomes uniformly small of order $v^{-(s+1)}$ (where $x=u+\sqrt{-1} v, u$ and $v$ real) as $v$ becomes infinite in the region, the representation is said to be asymptotic in that region with respect to $v$, for $|y|$ small.

In an entirely similar manner we define asymptotic representation of a function by a series of the type (3) with respect to $x$ or $v$, except that this time it is for $|y|$ large.

The following inequalities are of importance to us:

$$
\sum_{\nu=1}^{\infty} \frac{1}{x-\left.\nu\right|^{s}}<\frac{\pi}{2|x|^{o-1}}
$$


for $x$ in the left half of the plane, and

$$
\sum_{\nu=1}^{\infty} \frac{1}{|x-\nu|^{s}}<\frac{2}{|v|^{s-1}}\left[\frac{1}{|v|}+\frac{\pi}{2}\right]
$$

for $x$ in the right half of the plane.*

We are now ready to prove

THEOREM A. Form the product

$$
p_{m}(x, y)=p\left(x-1, \frac{y}{r}\right) p\left(x-2, \frac{y}{r^{2}}\right) \cdots p\left(x-m, \frac{y}{r^{m}}\right) t\left(x-m, \frac{y}{r^{m}}\right) \text {. }
$$

As $m$ becomes infinite this product converges, for $k$ sufficiently large, to a definite limit function $g(x, y)$ independent of $k$. The function $g(x, y)$ is analytic throughout the finite $x$-and $y$-planes; its asymptotic form is given by $s(x, y)$ with respect to $x$ in any left half of the $x$-plane, for $|y|$ small, and with respect to $v$ in any right half of the $x$-plane, for $|y|$ small; it constitutes a principal solution of the equation (1).

Proof. We may write

where

$$
p_{m}(x, y)=t(x, y) \bar{p}_{m}(x, y)
$$

$$
\begin{aligned}
\bar{p}_{m}(x, y)= & {\left[t^{-1}(x, y) p\left(x-1, \frac{y}{r}\right) t\left(x-1, \frac{y}{r}\right)\right] } \\
& {\left[t^{-1}\left(x-1, \frac{y}{r}\right) p\left(x-2, \frac{y}{r^{2}}\right) t\left(x-2, \frac{y}{r^{2}}\right)\right] } \\
& \cdots\left[t^{-1}\left(x-m+1, \frac{y}{r^{m-1}}\right) p\left(x-m, \frac{y}{r^{m}}\right) t\left(x-m, \frac{y}{r^{m}}\right)\right] .
\end{aligned}
$$

$t(x, y)$ is a polynomial or a series convergent in the vicinity of $(\infty, 0)$ (multiplied by certain factors, cf. (2)). In order to show that $p_{m}(x, y)$ converges to a limit function it will therefore suffice to prove that $\bar{p}_{m}(x, y)$ does. To this end our attention will now be directed.

We have expressed $\bar{p}_{m}(x, y)$ as the product of functions of the type

$$
t^{-1}(x+1, r y) p(x, y) t(x, y)=1+n(x, y),
$$

$n(x, y)$ being a power series in $1 / x$ and $y$ which converges in the vicinity of $(\infty, 0)$ and has no terms of degree less than $k$ in $1 / x$ and $y$. Let, first of all, $k$ be chosen greater than or equal to 2 ; then it may be regarded as the sum of two integers $l$ and $d$, each of which is at least equal to 1 ; i. e.,

$$
k=l+d,
$$

$l, d \geqq 1$.

* The proof of these inequalities is given in some detail by Birkhoff, loc. cit., pp. 248-249. 
During the proof of convergence $k, l$, and $d$ are all to be regarded as fixed. We may write

$$
\begin{aligned}
& \bar{p}_{m}(x, y)= {\left[1+n\left(x-1, \frac{y}{r}\right)\right]\left[1+n\left(x-2, \frac{y}{r^{2}}\right)\right] } \\
& \cdots\left[1+n\left(x-m, \frac{y}{r^{m}}\right)\right]=1+\sum_{k_{1}=1}^{m} n\left(x-k_{1}, \frac{y}{r^{k_{1}}}\right) \\
& \quad+\sum_{k_{1}=1}^{m-1} \sum_{k_{1}=k_{1}+1}^{m} n\left(x-k_{1}, \frac{y}{r^{k_{1}}}\right) n\left(x-k_{2}, \frac{y}{r^{k_{2}}}\right)+\cdots
\end{aligned}
$$

Now inasmuch as $n(x, y)$ is convergent for $(x, y)$ in the vicinity of $(\infty, 0)$, it follows that if $x$ lies in a certain region $D$, shown in the accompanying

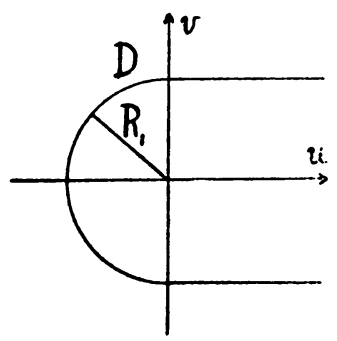

$X$-plane figure and such that $x-i(i=1,2,3, \ldots)$ lies outside the circle of radius $R_{1}$ about the origin, and $y$ lies inside an associated circle of radius $r_{2}$ about the origin in the $y$-plane, each of the $n$-series occurring in (6) will be convergent. For the present it will be supposed that $x$ and $y$ lie in these regions of their respective planes.

In order to prove the convergence of the multiple series which (6) becomes upon allowing $m$ to increase without limit we shall break each function $n\left(x-k_{i}, y / r^{k_{i}}\right)$ into two parts, the first consisting of all the terms in which $y$ occurs to a power $\geqq l$ and the second made up of the remaining terms. For places $(x, y)$ in the region specified the first part is less in absolute value than $\bar{M} /|\boldsymbol{r}|^{k_{i} l}$ and the second is less in absolute value than $\bar{M} /\left|x-k_{i}\right|^{d+1}$, where $\bar{M}$ and $\bar{M}$ are certain positive constants independent of the value of $k_{i}$. The terms of (6), omitting the first, are therefore less in absolute value than the corresponding terms of the following series:

$$
\begin{aligned}
\sum_{k_{1}=1}^{m}\left[\frac{\bar{M}}{|r|^{k_{2} l}}+\frac{\bar{M}}{\left|x-k_{1}\right|^{d+1}}\right]+\sum_{k_{1}=1}^{m-1} \sum_{k_{r}=k_{1}+1}^{m}\left[\frac{\bar{M}}{|r|^{k_{1} l}}+\frac{\bar{M}}{\left|x-k_{1}\right|^{d+1}}\right] \\
\cdot\left[\frac{\bar{M}}{|r|^{k_{2} l}}+\frac{\bar{M}}{\left|x-k_{2}\right|^{d+1}}\right]+\cdots
\end{aligned}
$$

As $m$ becomes infinite this sum approaches the limit

$$
\begin{aligned}
{\left[\left(1+\frac{\bar{M}}{|r|^{2}}+\frac{\bar{M}}{|x-1|^{d+1}}\right)\right.} & \left(1+\frac{\bar{M}}{|r|^{2 l}}+\frac{\bar{M}}{|x-2|^{d+1}}\right) \\
& \left.\cdot\left(1+\frac{\bar{M}}{|r|^{3 l}}+\frac{\bar{M}}{|x-3|^{d+1}}\right) \ldots\right]-1
\end{aligned}
$$


Since $|r|$ is $>1$ and both $l$ and $d$ are $\geqq 1$, the bracketed infinite product converges. It follows that $\bar{p}_{m}(x, y)$, and therefore $p_{m}(x, y)$, converges absolutely and uniformly to a function analytic in the neighborhood of places $(x, y)$ for which $x$ is in $D$ and $|y|$ is $<r_{2}$. These limit functions we denote respectively by $\bar{g}(x, y)$ and $g(x, y)$.

Inasmuch as it is possible to write

$$
p_{m}(x, y)=p\left(x-1, \frac{y}{r}\right) p\left(x-2, \frac{y}{r^{2}}\right) \cdots p\left(x-h, \frac{y}{r^{h}}\right) p_{m-h}\left(x-h, \frac{y}{r^{h}}\right)
$$

and to give the same argument for $p_{m-h}\left(x-h, y / r^{h}\right)$ as has been given above for $p_{m}(x, y), h$ being arbitrarily large, we conclude that $p_{m}(x, y)$ converges also for places $(x, y)$ for which $x$ is finite but not in $D$ or $|y|$ is finite but $>r_{2}$, or both these conditions obtain. This completes the proof of convergence.

The function $g(x, y)$ is independent of $k$, because if $k^{\prime}$ is any second value for $k$ and $t^{\prime}(x, y)$ the function corresponding to $t(x, y)$, we may write

$$
\begin{aligned}
p_{m}^{\prime}(x, y)=t(x, y) & {\left[t^{-1}(x, y) p\left(x-1, \frac{y}{r}\right) t\left(x-1, \frac{y}{r}\right)\right] } \\
\cdot & {\left[t^{-1}\left(x-1, \frac{y}{r}\right) p\left(x-2, \frac{y}{r^{2}}\right) t\left(x-2, \frac{y}{r^{2}}\right)\right] } \\
\cdots & {\left[t^{-1}\left(x-m+1, \frac{y}{r^{m-1}}\right) p\left(x-m, \frac{y}{r^{m}}\right) t^{\prime}\left(x-m, \frac{y}{r^{m}}\right)\right] . }
\end{aligned}
$$

Then if $p_{m}^{\prime}(x, y)$ be expanded in a sum like (6) and $m$ be allowed to become infinite, the resulting multiple series for $g(x, y)$ will be term by term identical with that obtained above.

We have still to examine the asymptotic form of $g(x, y)$. First of all let it be observed that the expression (7) is less than

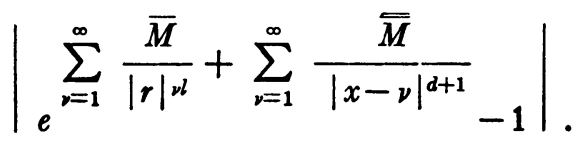

Hence the limit function $\bar{g}(x, y)$ differs from 1 by a quantity less in absolute value than

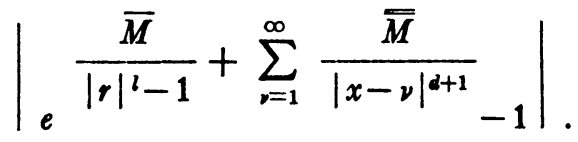


By taking $k$, and with it $l$, sufficiently large, however, we can make the first term in the exponent of $e$ as small as we please. Yet it has been shown that $\bar{g}(x, y)$ is independent of $k$. It therefore follows that we have

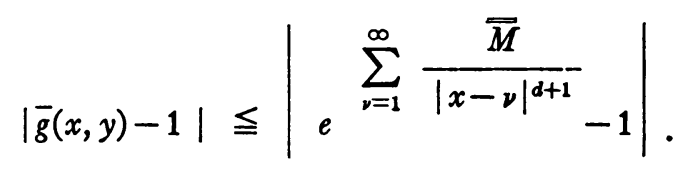

By virtue of (4) if $x$ lies in any left half-plane, the expression inclosed in bars on the right has the form $M(x) / x^{d}$ where $M(x)$ is a bounded function. Thus for $x$ in $D$ and in any left half-plane, and for $y$ inside the circle of radius $r_{2}$ about the origin, we have

$$
\bar{g}(x, y)=1+\frac{M(x, y)}{x^{d}}, \quad \quad|M(x, y)|<M,
$$

$M$ being a certain positive number. Similarly, for $x$ in $D$ and in any right half-plane and for $|y|<r_{2}$, we have by (5)

$$
\bar{g}(x, y)=1+\frac{M^{\prime}(x, y)}{v^{d}}, \quad \quad\left|M^{\prime}(x, y)\right|<M^{\prime},
$$

where $M^{\prime}$ is a positive constant. Using the first of these expressions for $\bar{g}(x, y)$ we find

$$
\begin{aligned}
g(x, y)=t(x, y) \bar{g}(x, y) & =t(x, y)+t(x, y) \frac{M(x, y)}{x^{d}} \\
=x^{\mu x} e^{-\mu x} x^{a} y p & \left\{\left(s+\frac{s_{10}}{x}+s_{01} y+\cdots\right)\right. \\
+ & \left.+\frac{M(x, y)}{x^{d}}\left(s+\frac{s_{10}}{x}+s_{01} y+\cdots\right)\right\} .
\end{aligned}
$$

Now if the series (or polynomial) contained in the first parentheses within the braces be broken off so as to retain only those terms in which the exponent of $1 / x$ is $s$ or less and that of $y$ is $t$ or less, the remainder may be regarded as consisting of two pieces, one of which is infinitesimal in $x$ to the order $s+1$ and the other infinitesimal in $y$ to the order $t+1$. At the same time the second term within the braces can be made infinitesimal in $x$ to the order $s+1$ by taking $k$, and with it $d$, sufficiently large. The reasoning and result are similar if we use the second of the above expressions for $\bar{g}(x, y)$. Hence $g(x, y)$ is represented asymptotically by $s(x, y)$ with 
respect to $x$ in any left half-plane and with respect to $v$ in any right halfplane, for $|y|$ small.

It remains for us to justify the term principal solution which has been applied to the function $g(x, y)$. To do this it will suffice to show that the properties which this solution possesses characterize it completely. Let it be supposed, then, that there exists a second solution $g^{\prime}(x, y)$ of (1) which possesses the same properties as $g(x, y)$. The quotient $g(x, y) / g^{\prime}(x, y)=a(x, y)$ is clearly an analytic function except perhaps for poles over the entire finite $x$ - and $y$-planes; furthermore it is periodic in such a way that $a(x+1, r y)=$ $a(x, y)$. Consider any place $\left(x_{0}, y_{0}\right)$ for which $x_{0}$ and $y_{0}$ are finite; the value of the quotient $a(x, y)$ at this place is the same as its value at $\left(x_{0}-1, y_{0} / r\right)$, and at $\left(x_{0}-2, y_{0} / r^{2}\right)$, etc. But inasmuch as $g(x, y)$ and $g^{\prime}(x, y)$ both have the asymptotic form (2) for $x$ in any left half-plane and $|y|$ small, the quotient $a(x, y)$ approaches the value 1 as $(x, y)$ traverses the sequence $\left(x_{0}, y_{0}\right),\left(x_{0}-1, y_{0} / r\right),\left(x_{0}-2, y_{0} / r^{2}\right), \cdots$. It follows that $a\left(x_{0}, y_{0}\right)$ is itself 1 , and in consequence we have $g(x, y) \equiv g^{\prime}(x, y)$.

Using the methods which have been employed above we can establish a second theorem. For this purpose it is necessary that we define a function $t^{\prime}(x, y)$ which bears the same relation to $s^{\prime}(x, y)$ that $t(x, y)$ does to $s(x, y)$; i. e., $t^{\prime}(x, y)$ is a series convergent in the vicinity of $(\infty, \infty)$ and having the same terms as $s^{\prime}(x, y)$ up to and including those of degree $k-1$ in $1 / x$ and $1 / y$ together. The statement of the theorem follows.

TheOREM B. Form the product

$$
p_{m}^{\prime}(x, y)=p^{-1}(x, y) p^{-1}(x+1, r y) \cdots p^{-1}\left(x+m-1, r^{m-1} y\right) t^{\prime}\left(x+m, r^{m} y\right) .
$$

As $m$ becomes infinite this product converges, for $k$ sufficiently large, to a definite limit function $h(x, y)$, independent of $k$. The function $h(x, y)$ is analytic throughout the finite $x$-plane and the extended $y$-plane with the exception of $y=0$, and except at places which are poles of $p^{-1}(x, y)$, or of $p^{-1}(x+1, r y)$, or ...; at these places the function $h(x, y)$ itself has poles. The asymptotic form of $h(x, y)$ is given by $s^{\prime}(x, y)$ with respect to $x$ in any right half of the $x$-plane, for $|y|$ large, and with respect to $v$ in any left half of the $x$-plane, for $|y|$ large. The function $h(x, y)$ constitutes a second principal solution of the equation (1).

The relation between the solutions. If we define the function $a(x, y)$ by means of the equation

$$
g(x, y)=h(x, y) a(x, y)
$$

it is clear that $a(x, y)$ is periodic in such a fashion that $a(x+1, r y)=a(x, y)$. It is also evident that $a(x, y)$ is analytic except possibly for poles over the 
region consisting of the entire finite $x$-plane and of the $y$-plane between circles $r_{2}$ and $R_{2}$ about the origin, where $r_{2}$ is arbitrarily small and $R_{2}$ arbitrarily large. That no such poles can actually occur, however, is easily demonstrated by methods already employed elsewhere* by the writer. A further analysis of the periodic function $a(x, y)$ seems feasible only in a very restricted case, and we shall not attempt it here.

\section{A MORE GENERAL PROBLEM}

The seeming lack of symmetry in the formal series solutions (2) and (3) is only apparent, for if we had begun by supposing that in the polynomial $p(x, y)$ the lowest power of $y$ to occur is $y^{\lambda}$ and that $p_{\mu \lambda}$ is different from zero, we should have had in place of (2) the formal solution

$$
s(x, y)=x^{\mu x} e^{-\mu x} r^{\lambda\left(r^{2}-\tau\right) / 2} x^{a} y^{p}\left[s+\frac{s_{10}}{x}+s_{01} y+\cdots\right] .
$$

After this remark it becomes clear that $p(x, y)$ may just as well be taken to be a polynomial in $x, 1 / x, y$, and $1 / y$, it being essential only that $p(x, y)$ be expressible in the forms

$x^{\mu} y^{\lambda}$ [polynomial in $1 / x$ and $y$ with constant term $\neq 0$ ],

$x^{\mu} y^{\nu}$ [polynomial in $1 / x$ and $1 / y$ with constant term $\neq 0$ ].

Furthermore it may be observed that formal series solutions can be found and existence theorems analogous to those of $\$ 1$ proved as above if the coefficient function in (1) is any function whatever expressible in each of the following forms, the first valid in the vicinity of $(\infty, 0)$ and the second valid in the vicinity of $(\infty, \infty)$ :

$x^{\mu} y^{\lambda}$ [convergent power series in $1 / x$ and $y$ with constant term $\neq 0$ ],

$x^{\mu} y^{\nu}$ [convergent power series in $1 / x$ and $1 / y$ with constant term $\neq 0$ ]. The only difference in the results is that the solutions obtained may be meromorphic only in limited regions about $(\infty, 0)$ and $(\infty, \infty)$ respectively.

* Cf. I, pp. 304-305.

BROWN UNIVERSITY, Providence, R. I. 